\title{
In-situ Liquid Electrochemical TEM Investigation of Semi Solid-State LMNO Micro- Battery
}

Ankush Bhatia ${ }^{1}$, Maxime Hallot ${ }^{2}$, Sorina Cretu ${ }^{3}$, Nicolas Folastre ${ }^{4}$, Maxime Berthe ${ }^{5}$, David Troadec $^{5}$, Pascal Roussel $^{6}$, Jean-Pierre Pereira-Ramos ${ }^{7}$, Rita Baddour-Hadjean ${ }^{7}$, Christophe Lethien ${ }^{5}$ and Arnaud Demortière $^{4}$

${ }^{1}$ Laboratoire de Réactivité et de Chimie des solides (LRCS), Université de Picardie Jules Verne, CNRS UMR 7314, 33 rue Saint Leu, 80039 Amiens Cedex, France, Thiais, France, ${ }^{2}$ Institut d'Electronique, de Microélectronique et de Nanotechnologies, Université de Lille,Centrale Lille,ISEN, Université de Valenciennes, CNRS UMR 8520- IEMN, F-59000 Lille, France, Languedoc-Roussillon, France, ${ }^{3}$ Laboratoire de Réactivité et de Chimie des solides (LRCS), Université de Picardie Jules Verne, CNRS UMR 7314, 33 rue Saint Leu, 80039 Amiens Cedex, France, France, ${ }^{4}$ Laboratoire de Réactivité et de Chimie des solides (LRCS), Université de Picardie Jules Verne, CNRS UMR 7314, 33 rue Saint Leu, 80039 Amiens Cedex, France, United States, ${ }^{5}$ Institut d'Electronique, de Microélectronique et de Nanotechnologies, Université de Lille,Centrale Lille,ISEN, Université de Valenciennes, CNRS UMR 8520- IEMN, F-59000 Lille, France, United States, ${ }^{6}$ Unité de Catalyse et de Chimie du Solide (UCCS), Université de Lille, CNRS, Centrale Lille, ENSCL, Université d'Artois, UMR 8181 - UCCS, F-59000 Lille, France, United States, ${ }^{7}$ Institut de Chimie et des Matériaux Paris Est (ICMPE), CNRS UMR 7182 -Université Paris Est Créteil, 2-8 rue Henri Dunant, 94320 Thiais, France, United States

All-solid-state Li-ion micro-battery is a promising candidate to power miniaturized sensors for the Internet of things (IoT) and other electronic devices. In recent times, the spinel $\mathrm{LiMn}_{1.5} \mathrm{Ni}_{0.5} \mathrm{O}_{4}$ (LMNO) has demonstrated as a potential positive electrode material for $\mathrm{Li}$-ion thin-film batteries offering a theoretical capacity of $147 \mathrm{mAh} / \mathrm{g}\left(65 \mu \mathrm{Ah} / \mathrm{cm}^{2} / \mu \mathrm{m}\right.$ for a bulk density of $\left.4.47 \mathrm{~g} / \mathrm{cm}^{3}\right)$ and operates up to now at the highest potential (around $4.7 \mathrm{~V}$ vs. $\mathrm{Li}^{+} / \mathrm{Li}$ ) [1]. In this work, we report our first successful in situ TEM attempts to observe the morphological, structural, and interfacial changes in the positive electrode layer of FIB prepared sample which undergo after cycling using liquid electrolyte [2-3]. More precisely we compared the morphological and structural evolution between a pristine and cycled micro battery by 4D STEM-ASTAR technique to highlight the key information to improve the deposition conditions that will enhance the reliability and production quality of such micropower devices.

Here, our approach is based on cycling a FIB lamella sample inside the TEM using a liquidelectrochemical TEM holder with conventional liquid electrolyte $\left(1 \mathrm{M} \mathrm{LiClO}_{4}, \mathrm{EC}\right.$ : DMC 1:1). The crosssection image of as-prepared FIB sample with homogeneous deposition of distinctive layers of different thickness [from bottom to top-Si $(0.385 \mathrm{~mm}) / \mathrm{Al}_{2} \mathrm{O}_{3}(100 \mathrm{~nm}) / \mathrm{Pt}(630 \mathrm{~nm}) / \mathrm{LNMO}(400 \mathrm{~nm})$ ] is shown in figure 1a. The Pt current collector of the FIB lamella sample is connected to the Pt working electrode on the e-chips used for the TEM study (figure 1b). First, using the FIB preparation technique, we sliced a full 2-D "thin-film micro-battery" making it as thin to observe/analyze under TEM. Then, we modified the FIB lamellar design using the FIB-SEM tool to get good electrical contact and reduced polarisation. Several technological problems have to be overcome in the process. For the instance, it is mandatory to obtain good electrical contact between the Pt working electrode of the e-chip and Pt current collector of FIB lamellar, which is later achieved by depositing extra Pt between the two contacts forming a platinum bridge. A 4-Probe electrical conductivity performed locally confirms the good electrical contact between 
the as prepared FIB lamella sample and Pt working electrode of e-chip. The cross-section bright-field TEM image of a final modified version of the FIB lamella sample used in the study is shown in figure $2 \mathrm{a}$.

The FIB lamella sample is then later cycled inside the liquid electrochemical TEM holder (fig 1c) in a potential window of $4.1 \mathrm{~V}-4.8 \mathrm{~V}$ vs. $\mathrm{Li}^{+} / \mathrm{Li}$. The flow of the electrolyte (LiClO4 EC: DMC 1:1) inside the TEM holder was further controlled by the microfluidic controller with a flow rate of $2 \mu \mathrm{L} / \mathrm{min}$. CV was recorded at a sweep rate of $0.1 \mathrm{mV} / \mathrm{s}$ and two plateaus at $4.4 \mathrm{~V}$ and $4.6 \mathrm{~V}$ were observed corresponding to $\mathrm{Ni}^{2+/ 3+}$ and $\mathrm{Ni}^{3+/ 4+}$ oxidation respectively (Fig $2 \mathrm{c}$ ). The basic redox steps observed during the charge are same as observed in the case of cycling bulk 2D thin film in a homemade flat cell.

The comparison between the cycled and pristine micro battery sliced by FIB and observed by TEM allowed us to clearly demonstrate the formation of cracks inside the LMNO layer, loss of contact between the LMNO layer and the platinum current collector, as well as the agglomeration of the organic compounds produced due to the electrolyte decomposition. Moreover, the 4D STEM-ASTAR technique [4] provided us with crucial information regarding the grain size reduction from $20 \mathrm{~nm}$ in pristine to 12 $\mathrm{nm}$ in the cycled sample, confirming the continuous electrode-electrolyte reaction happening over the cycling. Also, the decrease in crystallinity and increase in the amorphization of the LMNO grains by 38 $\%$ in the cycled sample compared to pristine as shown in figure $2 b$ and $2 d$ clearly prove the fast capacity fading phenomenon observed in the bulk micro battery. Furthermore, a thickness of 20nm along the platinum layer, a (111) preferred orientation is observed exhibiting the epitaxial effect of LMNO on the platinum layer which has been further supported by the precision electron diffraction (PED) recorded on both LMNO and platinum grains.

Acknowledgments

Ankush Bhatia and Arnaud Demortière acknowledges the French ANR project "CASSIOPES"

N¹7-CE09-0016-03 for a Ph.D. grant. We would like to thank Dr. Muriel Veron and Dr. Edgar Rauch for the fruitful discussions on the ASTAR experiments.
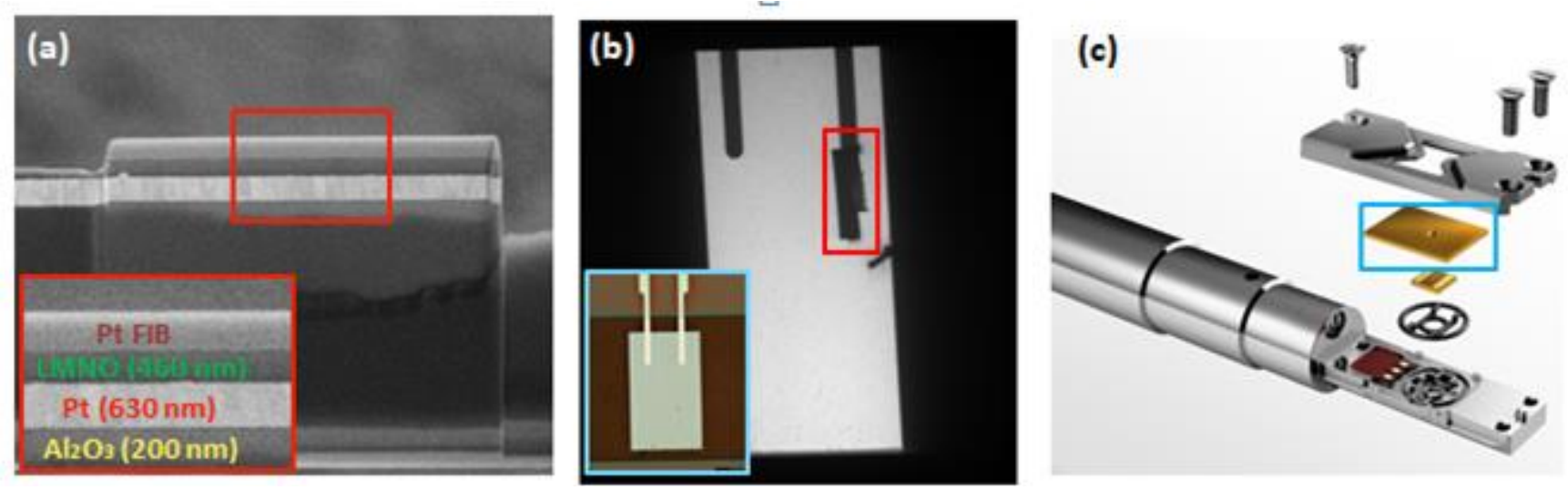

Figure 1. (a) Cross-section image of as-prepared FIB sample with homogeneous deposition of distinctive layers of different thickness, insert in the red box shows the zoom of the different layers; (b) TEM image exhibiting the connection between the platinum current collector of FIB sample with the platinum working electrode of e-chip; insert figure shows the reference and the working electrode on the silicon nitride window; (c) schematic description of the Poseidon 510 TEM holder ( Protochips) for electrochemical measurements used in this study. 

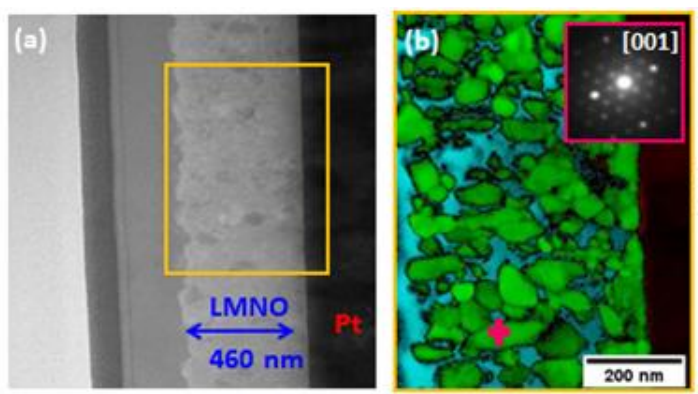

Before Cycling
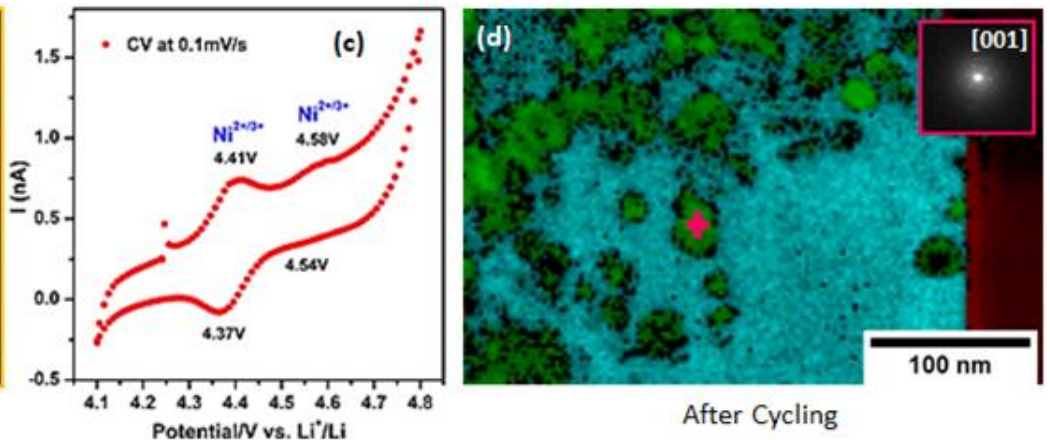

Figure 2. (a) Bright-field transmission electron microscopy of the LMNO FIB lamella sample before cycling; (b) phase map before cycling where dark red represents the platinum, in green the LMNO phase, and in blue the amorphous phase; insert bright red shows the electron diffraction pattern of spinel LMNO in [001] orientation; (c) Cyclic voltammogram measured in a holder with a voltage sweep rate of $0.1 \mathrm{mV} / \mathrm{s}$ in the voltage window of $4.1 \mathrm{~V}-4.8 \mathrm{~V}$ vs. lithium using $1 \mathrm{M}$ lithium perchlorate in EC:DMC electrolyte; (d) phase map after cycling where dark red represents the platinum, in green the LMNO phase and in blue the amorphous phase; insert bright red shows the electron diffraction pattern of spinel LMNO in [001] orientation.

\section{References}

[1] M. Hallot, A. Demortière, P. Roussel, C. Lethien, Energy Storage Materials, (18)30779-7

[2] O.M. Karakulina, A. Demortière, W. Dachraoui, A.M. Abakumov, J. Hadermann, Nano lett. 18(2018) 6286-6291.

[3] L. Lutz, W. Dachraoui, A. Demortière, L. R. Johnson, P. G. Bruce, A. Grimaud, J.M. Tarascon, Nano Lett. 18(2018), 1280-1289

[4] E. F. Rauch, M. Véron, Mater. Charact. 98 (2014), 1-9. 